\title{
OPERATOR SELF-SIMILAR PROCESSES ON BANACH SPACES
}

\author{
MIHAELA T. MATACHE AND VALENTIN MATACHE
}

Received 22 March 2005; Revised 21 July 2005; Accepted 30 July 2005

Operator self-similar (OSS) stochastic processes on arbitrary Banach spaces are considered. If the family of expectations of such a process is a spanning subset of the space, it is proved that the scaling family of operators of the process under consideration is a uniquely determined multiplicative group of operators. If the expectation-function of the process is continuous, it is proved that the expectations of the process have power-growth with exponent greater than or equal to 0 , that is, their norm is less than a nonnegative constant times such a power-function, provided that the linear space spanned by the expectations has category 2 (in the sense of Baire) in its closure. It is shown that OSS processes whose expectation-function is differentiable on an interval $\left(s_{0}, \infty\right)$, for some $s_{0} \geq 1$, have a unique scaling family of operators of the form $\left\{s^{H}: s>0\right\}$, if the expectations of the process span a dense linear subspace of category 2 . The existence of a scaling family of the form $\left\{s^{H}: s>0\right\}$ is proved for proper Hilbert space OSS processes with an Abelian scaling family of positive operators.

Copyright (c) 2006 M. T. Matache and V. Matache. This is an open access article distributed under the Creative Commons Attribution License, which permits unrestricted use, distribution, and reproduction in any medium, provided the original work is properly cited.

\section{Introduction}

Let $\mathscr{E}$ denote a Banach space and let $\mathscr{L}(\mathscr{E})$ be the algebra of all linear bounded operators on $\mathscr{E}$. Let $(\Omega, \mathscr{Y}, P)$ be a probability space. Throughout this paper, given a random variable $X: \Omega \rightarrow \mathscr{E}$, the measure $P X^{-1}$ denotes the distribution of $X$, that is, the following Borel probability measure:

$$
P X^{-1}(S)=P\left(X^{-1}(S)\right)
$$

The fact that two stochastic processes $\{X(t): t>0\}$ and $\{Y(t): t>0\}$ are identically distributed, that is, all their finite-dimensional distributions are equal, will be designated by the notation $\{X(t)\} \stackrel{d}{=}\{Y(t)\}$. 
Definition 1.1. An operator self-similar process is a stochastic process $\{X(t): t>0\}$ on $\mathscr{E}$ with a scaling family of operators $\{A(s): s>0\}$, that is, a process such that there is a family $\{A(s): s>0\}$ in $\mathscr{L}(\mathscr{E})$ with the property that for each $s>0$,

$$
\{X(s t)\} \stackrel{d}{=}\{A(s) X(t)\}
$$

The property above will be referred to as the self-similarity of the process $\{X(t): t>0\}$ under the scaling family of operators $\{A(s): s>0\}$. The term operator self-similar will be designated by the acronym OSS throughout this paper. If the process has a scaling family of the particular form

$$
A(s)=s^{H} I, \quad s>0,
$$

where $H$ is some fixed scalar and $I$ denotes the identity operator, then it is called selfsimilar instead of OSS.

Self-similar processes were introduced by Lamperti, in 1962 [10]. OSS processes appeared later [9]. In this paper we consider and study OSS processes valued in (possibly infinite-dimensional) Banach spaces. Our main idea is to obtain information about such processes by using the theory of one-parameter semigroups and groups of operators (see Definitions 1.2 and 1.3 below for these notions).

This section is dedicated to summarizing the main results, introducing the basic notions, setting up notation, and giving some examples. Examples 1.4 and 1.9 in this section emphasize why it is natural to think of groups of operators in connection with OSS processes. In Section 2 we study OSS processes with rich families of expectations. Theorem 2.3 in that section says that, if the linear space spanned by the expectations of such a process is a set having category 2 in the sense of Baire in its closure, then there exist constants $a \geq 0$ and $M \geq 1$ such that

$$
\begin{aligned}
& \|E[X(t)]\| \leq M t^{a}, \quad t \geq 1, \\
& \|E[X(t)]\| \leq M t^{-a}, \quad 0<t \leq 1 .
\end{aligned}
$$

In order that the inequalities above hold we also require that the OSS processes have a norm-continuous expectation-function $t \rightarrow E(X(t)), t>0$. The main ingredient in the proof of the theorem is the fact that OSS processes whose expectations span a dense linear subspace of the whole space have a unique scaling family of operators which is necessarily a multiplicative group of operators. This is proved in Theorem 2.1 of Section 2 and we say that such processes have a spanning family of expectations. For those processes having expectation-function, differentiable on an interval of the form $\left(s_{0}, \infty\right), s_{0} \geq 1$ we are able to show that the scaling family is necessarily of the form $\left\{s^{H}: s>0\right\}$ for some $H \in \mathscr{L}(\mathscr{E})$, (Theorem 2.4, Section 2).

In Section 3 we consider OSS processes with scaling families of invertible operators. The main result in that section is Theorem 3.5 which states that proper Hilbert-space 
valued OSS processes with a scaling family of commuting, invertible, positive operators have an exponent, that is, have a scaling family of operators of the form $\left\{s^{H}: s>0\right\}$ for some $H \in \mathscr{L}(\mathscr{E})$. For the term proper OSS process we refer the reader to Definition 3.5 in Section 3.

In order to set up terminology we recall the following definition.

Definition 1.2. An additive semigroup of operators on $\mathscr{E}$ is a family $\{T(t): t \geq 0\}, T(t) \in$ $\mathscr{L}(\mathscr{E})$, for all $t \geq 0$, with the following properties:

$$
T(0)=I, \quad T(s+t)=T(s) T(t), \quad \forall t, s \geq 0 .
$$

If one can define $T(t)$ for $t<0$ such that relation (1.5) holds for all $t, s \in \mathbb{R}$, then we say that $\{T(t): t \in \mathbb{R}\}$ is an additive group of operators. The theory of semigroups of operators is customarily exposed in "additive notation" [7, 14]. It can be easily translated into multiplicative notation as follows.

Definition 1.3. A multiplicative semigroup of operators on $\mathscr{E}$ is a family $\{A(s): s \geq 1\}$, $A(s) \in \mathscr{L}(\mathscr{E})$ with the following properties:

$$
A(1)=I, \quad A(s t)=A(s) A(t), \quad \forall t, s \geq 1 .
$$

If one can define $A(s)$ for $0<s<1$ such that relation (1.6) holds for all $t, s \in(0, \infty)$, then we say that $\{A(s): s>0\}$ is a multiplicative group of operators. It is easy to see that if $\{T(t)\}$ is an additive semigroup (group) of operators, then $\{A(t)\}$ given by $A(t):=$ $T(\log t)$ is a multiplicative semigroup (group) of operators and conversely, if $\{A(t)\}$ is a multiplicative semigroup (group) of operators, then $\{T(t)\}$ given by $T(t):=A\left(e^{t}\right)$ is an additive semigroup (group) of operators. For the purposes of this paper multiplicative notation is preferred and results taken from the theory of semigroups of operators and traditionally exposed in additive notation will be used in their multiplicative version by the mechanism exposed above. The reason for this choice is visible in Examples 1.4 and 1.9 .

Example 1.4. Consider a fixed random variable $X$ and a multiplicative group of operators $\{A(s): s>0\}$. Assume $\mathscr{E}$ is separable. For each $t>0$ let $\mu_{t}=P(A(t) X)^{-1}$. Consider each $n$ tuple $\{t\}=\left\{t_{1}, t_{2}, \ldots, t_{n}\right\}, t_{1}, t_{2}, \ldots, t_{n}>0$, and set $\mu_{\{t\}}:=\mu_{t_{1}} \times \mu_{t_{2}} \times \cdots \times \mu_{t_{n}}$. There exists a stochastic process $\{X(t): t>0\}$ whose finite-dimensional distributions are $\left\{\mu_{\{t\}}\right\}$. The process $\{X(t): t>0\}$ is an OSS process with scaling family of operators $\{A(s): s>0\}$.

Proof. It is easy to see that the family $\left\{\mu_{\{t\}}\right\}$, where we consider all possible ordered $n$ tuples $\{t\}, n=1,2, \ldots$, is a family of probability measures on $\mathscr{E}$ which obviously satisfies the consistency condition in Kolmogorov's extension theorem, [2, Theorem 4.4.3], thus there exists a stochastic process $\{X(t): t>0\}$ whose finite-dimensional distributions are $\left\{\mu_{\{t\}}\right\}$. The fact that this process is OSS with scaling family $\{A(s): s>0\}$ is a consequence of the fact that $\{A(s): s>0\}$ is a one-parameter, multiplicative group of operators. 
Indeed, for arbitrary, fixed $s>0$ and $\{t\}=\left\{t_{1}, t_{2}, \ldots, t_{n}\right\}, t_{1}, t_{2}, \ldots, t_{n}>0$, one can write

$$
\begin{aligned}
& P\left(\left\{X\left(s t_{1}\right) \in B_{1}, X\left(s t_{2}\right) \in B_{2}, \ldots, X\left(s t_{n}\right) \in B_{n}\right\}\right) \\
& \quad=\mu_{s\{t\}}\left(B_{1} \times B_{2} \times \cdots \times B_{n}\right)=\mu_{s t_{1}} \times \mu_{s t_{2}} \times \cdots \times \mu_{s t_{n}}\left(B_{1} \times B_{2} \times \cdots \times B_{n}\right) \\
& \quad=\prod_{j=1}^{n} P\left\{X\left(s t_{j}\right) \in B_{j}\right\}=\prod_{j=1}^{n} P\left\{A\left(s t_{j}\right) X \in B_{j}\right\} \\
& \quad=\prod_{j=1}^{n} P\left\{A(s) A\left(t_{j}\right) X \in B_{j}\right\}=\prod_{j=1}^{n} P\left\{A\left(t_{j}\right) X \in A(s)^{-1}\left(B_{j}\right)\right\} \\
& \quad=P\left(\left\{A(s) X\left(t_{1}\right) \in B_{1}, A(s) X\left(t_{2}\right) \in B_{2}, \ldots, A(s) X\left(t_{n}\right) \in B_{n}\right\}\right),
\end{aligned}
$$

for arbitrarily chosen Borel subsets $B_{1}, \ldots, B_{n}$.

Another source of examples is the following generalization of a construction by Lamperti.

Definition 1.5. Let $\{Y(t): t \in \mathbb{R}\}$ be an $\mathscr{E}$-valued stochastic process and $\{A(s): s>0\}$ a family of bounded linear operators on $\mathscr{E}$ with the property

$$
A(s t)=A(s) A(t) \quad \forall s, t>0 .
$$

The process

$$
X(t):=A(t) Y(\log t), \quad t>0,
$$

is called the Lamperti transform of the process $\{Y(t): t \in \mathbb{R}\}$ under the scaling family of operators $\{A(t): t>0\}$.

To see how the Lamperti transform is a source of examples of OSS processes, consider the following.

Example 1.6. The Lamperti transform of a strictly stationary process under a scaling family of operators with the property in Definition 1.5 is an OSS process.

Proof. Indeed observe that for arbitrary, fixed $s>0$ one can write

$$
\{X(s t)\}=\{A(s t) Y(\log s+\log t)\} \stackrel{d}{=}\{A(s) A(t) Y(\log t)\}=\{A(s) X(t)\}
$$

so $\{X(t): t>0\}$ is OSS.

It is worth observing that a converse construction is also true.

Remark 1.7. If $\{X(t): t>0\}$ is an OSS process having a scaling family of operators $\{A(s)$ : $s>0\}$ which is a multiplicative group of operators, then the process

$$
Y(t)=A\left(e^{t}\right)^{-1} X\left(e^{t}\right), \quad t \in \mathbb{R}
$$

is strictly stationary and has Lamperti transform under the scaling family $\{A(s): s>0\}$ equal to $\{X(t): t>0\}$. 
Proof. Indeed for arbitrary, fixed $h \in \mathbb{R}$ one has

$$
\begin{aligned}
\{Y(t+h)\} & =\left\{A\left(e^{h} e^{t}\right)^{-1} X\left(e^{h} e^{t}\right)\right\} \stackrel{d}{=}\left\{A\left(e^{t}\right)^{-1} A\left(e^{h}\right)^{-1} A\left(e^{h}\right) X\left(e^{t}\right)\right\} \\
& =\left\{A\left(e^{t}\right)^{-1} X\left(e^{t}\right)\right\}=\{Y(t)\}
\end{aligned}
$$

so the process $\{Y(t): t>0\}$ is strictly stationary. Checking that its Lamperti transform under the scaling family $\{A(s): s>0\}$ equals $\{X(t): t>0\}$ is straightforward.

For $\mathbb{R}^{n}$-valued OSS processes, a connection between this class of processes and operator-stable probability measures has been observed by the authors of [4]. Their idea extends to Banach space valued processes as follows.

Example 1.8. Let $\mathscr{E}$ be a separable real Banach space and $\{A(s): s>0\}$ a multiplicative strongly continuous group of operators on $\mathscr{E}$. Let $\mu$ be a probability measure with a representation of the following form:

$$
\mu=\lim _{n \rightarrow \infty} A\left(s_{n}\right) \nu^{n} * \delta_{x_{n}}
$$

where the limit above should be taken in the sense of the weak convergence of probability measures, the powers $v^{n}$ of the probability measure $v$ are calculated with respect to the operation $*$ of convolution, $\left(s_{n}\right)$ is a sequence in $(0, \infty)$ and $\left(x_{n}\right)$ a sequence in $\mathscr{E}$. For each such $\mu$ there is a family $\left\{b_{t}: t>0\right\}$ of vectors in $\mathscr{E}$ such that setting for each $t>0$,

$$
\mu_{t}:=\mu^{t} * \delta_{b_{t}}
$$

and for each $n$-tuple $\{t\}=\left\{t_{1}, t_{2}, \ldots, t_{n}\right\}, t_{1}, t_{2}, \ldots, t_{n}>0, \mu_{\{t\}}:=\mu_{t_{1}} \times \mu_{t_{2}} \times \cdots \times \mu_{t_{n}}$, there exists a stochastic process $\{X(t): t>0\}$ whose finite-dimensional distributions are $\left\{\mu_{\{t\}}\right\}$. The process $\{X(t): t>0\}$ is OSS and $P(X(1))^{-1}=\mu$.

Proof. According to [5] such a measure is infinitely divisible and hence $\mu^{t}$ above makes sense for all $t>0$. Furthermore, according to the same paper, there exist $\alpha>0$ and a subset $\left\{b_{t}: t>0\right\}$ of $\mathscr{E}$ such that

$$
\mu^{t}=A\left(t^{\alpha}\right) \mu * \delta_{-b_{t}}
$$

[5, Theorem 3.2]. Arguing like in Example 1.4, one gets that, by Kolmogorov's extension theorem, there exists a process $\{X(t): t>0\}$ whose finite-dimensional distributions are $\mu_{\{t\}}$. That process is OSS with scaling family of operators $\left\{B(t)=A\left(t^{\alpha}\right): t>0\right\}$.

Of course, simpler examples of OSS processes can be given as well. For instance, we have the following examples.

Example 1.9. Given a fixed random variable $X$ and a one parameter, multiplicative group of operators $\{A(s): s>0\}$, the process $X(t):=A(t) X, t>0$ is OSS, with scaling family of operators $\{A(s): s>0\}$.

Lévy processes are random processes $\{X(t): t \geq 0\}$ which are stochastically continuous (i.e., for each $\epsilon>0, P(\{\|X(s+t)-X(s)\|>\epsilon\}) \rightarrow 0$ as $t \rightarrow 0)$, start almost surely at the 
origin (i.e., $X(0)=0$ a.s.), are time homogeneous (i.e., the distribution of $\{X(s+t)-$ $X(s): t \geq 0\}$ does not depend on $s$ ), have independent increments (i.e., $X\left(t_{0}\right), X\left(t_{1}\right)-$ $X\left(t_{0}\right), \ldots, X\left(t_{n}\right)-X\left(t_{n-1}\right)$, are independent for any choice $\left.0 \leq t_{0}<t_{1}<\cdots<t_{n}\right)$, and have continuous sample-paths.

Example 1.10. An important class of examples of OSS processes $\{X(t): t \geq 0\}$ are the proper Lévy processes having stationary, independent, operator-stable increments and the property that $X(1)$ has null centering function.

We refer the reader to [4, Theorem 7] for a proof and include in the following some explanations on the notions in the previous example. Operator-stable measures are measures obtained as limits, like the measure $\mu$ in Example 1.8. Such measures, on arbitrary Banach spaces, are studied in $[8,18-20]$. The fact that the class of operator-stable probability measures on a separable, infinite-dimensional Banach space and that the class of infinitely divisible laws coincide is established in [19]. In $[8,18]$ the existence of exponents for full (i.e., not supported on a hyperplane), operator-stable, probability measures is proved. This means that a full probability measure $\mu$ on a real, separable Banach space is proved to be operator-stable if and only if there is some operator $B$ so that $\mu^{t}$ is a translation of the measure $t^{B} \mu=e^{\log t B} \mu$, for each $t>0$. More formally, there is a function $t \rightarrow b_{t}$ called the centering function of $\mu$ such that $\mu^{t}=t^{B} \mu * \delta_{b_{t}}$, for all $t>0$.

The simple, yet important case of the joint limiting distribution of the sample moments $\sum_{i=1}^{n} X_{i}^{k}, 1 \leq k \leq m<\infty$, of a sequence $X_{i}: i \geq 1$ of $\mathbb{R}$-valued independent and identically distributed (i.i.d) random variables is considered in [11]. If $X_{1}, X_{2}, \ldots$ are i.i.d as $X$, then $X$ is said to belong to the domain of attraction of $Y$ if for some sequences $\left\{a_{n}\right\}$ and $\left\{b_{n}\right\}$ of scalars, $a_{n}^{-1}\left(X_{1}+X_{2}+\cdots+X_{n}-b_{n}\right) \rightarrow Y$. The authors of [11] show, among other things, that the random vector $Z=\left(X, X^{2}, X^{3}, \ldots\right)$ belongs to the generalized domain of attraction of some operator-stable law on $\mathbb{R}^{\infty}$ if and only if each $X^{k}$ belongs to the domain of attraction of some operator-stable law. The generalized domain of attraction of a random vector has a definition similar to that (given above) of the domain of attraction of a random variable, only that one substitutes the sequence $\left\{a_{n}^{-1}\right\}$ by a sequence of invertible operators and $\left\{b_{n}\right\}$ by a sequence of vectors. The results in [11] are summarized in the book [12, Chapter 10].

We considered important making the comments above relative to the existing literature on operator-stable laws on Banach spaces as those papers provide important context for this one.

We conclude the introductory part of this paper by noting that the definition used by some authors for both OSS processes and for self-similar processes is slightly different from ours. More exactly, a stochastic process $\{X(t): t>0\}$ is called OSS if for each $s>0$ there exist an operator $A(s)$ on $\mathscr{E}$ and $b(s) \in \mathscr{E}$ such that

$$
\{X(s t)\} \stackrel{d}{=}\{A(s) X(t)+b(s)\} .
$$

The function $s \rightarrow b(s)$ is called a drift-function. So in this paper we study OSS stochastic processes with null drift-function, and simply call them OSS processes. The same goes for self-similar processes. Also we do not include in the definition any continuity requirement and we consider the time interval to be $(0, \infty)$ rather than $[0, \infty)$. 


\section{OSS processes with a spanning set of expectations}

In this section the main idea is that OSS processes with a spanning set of expectations have uniquely determined scaling families of operators which are groups of operators. We prove this result and investigate its consequences. We begin by setting up some notations. For each subset $S$ of $\mathscr{E}, \operatorname{Span}(S)$ denotes the linear subspace of $\mathscr{E}$ spanned by the vectors in $S$ and $\overline{\operatorname{Span}}(S)$ the closure of that subspace.

A stochastic process $\{X(t): t>0\}$ is called integrable if

$$
\int_{\Omega}\|X(t)\| d P<\infty \quad \forall t>0
$$

In this case, for each $t>0, E[X(t)]$ denotes the expectation of the random variable $X(t)$.

Some popular examples of $\mathbb{R}^{n}$-valued OSS process have zero expectations (standard fractional brownian motions for instance). Since this is often a good assumption, the following remark is in order here.

Remark 2.1. Let $\{X(t): t>0\}$ be an OSS process and $\{A(s): s>0\}$ one of its scaling families of operators. If for each $t>0$ one sets $Y(t):=X(t)-E[X(t)]$ then the process $\{Y(t): t>0\}$ is OSS with scaling family $\{A(s): s>0\}$ and zero expectations.

Proof. Observe that for each $s, t>0$ one can write

$$
\begin{aligned}
A(s) E[X(t)] & =A(s) \int_{\Omega} X(t) d P \\
& =\int_{\Omega} A(s) X(t) d P=\int_{\Omega} X(s t) d P=E[X(s t)] .
\end{aligned}
$$

According to this computation one obtains

$$
\{A(s) Y(t)\}=\{A(s) X(t)-A(s) E[X(t)]\} \stackrel{d}{=}\{X(s t)-E[X(s t)]\}=\{Y(s t)\}
$$

which proves that $\{Y(t): t>0\}$ is OSS. Clearly $E[Y(t)]=0$, for all $t>0$.

Let us denote $\mathscr{E}_{0}=\overline{\operatorname{Span}}\{E[X(t)]: t>0\}$. This subspace has the following interesting properties.

Theorem 2.1. Let $\{X(t): t>0\}$ and $\mathscr{E}_{0}$ be as above. The subspace $\mathscr{E}_{0}$ is left invariant by any scaling family of operators $\{A(s): s>0\}$ under which $\{X(t): t>0\}$ is OSS, that is,

$$
A(s) \mathscr{E}_{0} \subseteq \mathscr{E}_{0} \quad \forall s>0
$$

For an arbitrary, fixed $y_{0} \in \mathscr{E}_{0}$ and each fixed $t>0$, set $Y(t):=X(t)+A(t) y_{0}$. The process $\{Y(t): t>0\}$ is OSS with scaling family $\{A(s): s>0\}$. If $\{X(t): t>0\}$ is an OSS process with a spanning family of expectations, that is, if $\mathscr{E}_{0}=\mathscr{E}$, then its scaling family of operators is a uniquely determined multiplicative group of operators.

Proof. The invariance property is an immediate consequence of the equality

$$
A(s) E[X(t)]=E[X(s t)] \quad \forall s, t>0,
$$


which was used and proved in detail in the proof of the previous remark. Observe that

$$
\{A(s) Y(t)\}=\left\{A(s) X(t)+A(s) A(t) y_{0}\right\} \stackrel{d}{=}\left\{X(s t)+A(s t) y_{0}\right\} \stackrel{d}{=}\{Y(s t)\} \quad \forall s>0,
$$

because for each fixed $s>0$ one can write

$$
A(s) A(t) E[X(u)]=E[X(s t u)]=A(s t) E[X(u)]
$$

which implies

$$
\begin{aligned}
& A(s) A(t) y_{0}=A(s t) y_{0}, \\
& \{A(s) X(t)\} \stackrel{d}{=}\{X(s t)\}
\end{aligned}
$$

by the operator self-similarity of $\{X(t): t>0\}$ under $\{A(s): s>0\}$. This proves that $\{Y(t): t>0\}$ is also OSS under the same scaling family of operators. To finish the proof, assume now that $\mathscr{E}_{0}=\mathscr{E}$ and that $\{A(s): s>0\}$ and $\{B(s): s>0\}$ are two scaling families of operators for $\{X(t): t>0\}$. For an arbitrary, fixed $s>0$ we wish to show that $A(s)=B(s)$, thus proving the uniqueness of the scaling family. This is an immediate consequence of the equalities

$$
A(s) E[X(t)]=E[X(s t)]=B(s) E[X(t)] \quad \forall s, t>0 .
$$

To show that $\{A(s): s>0\}$ is a multiplicative group of operators, observe that

$$
A(s t) E[X(u)]=E[X(s t u)]=A(s) A(t) E[X(u)], \quad \forall s, t, u>0,
$$

hence $A(s t)=A(s) A(t)$, for all $s, t>0$. Also

$$
A(1) E[X(u)]=E[X(u)]=I E[X(u)], \quad \forall u>0,
$$

hence $A(1)=I$.

Definition 2.2. An integrable process is said to have continuous expectation-function if the map

$$
t \longrightarrow E[X(t)], \quad t>0
$$

is norm-continuous.

Corollary 2.2. If $\{X(t): t>0\}$ is a scalar-valued integrable OSS process, with continuous expectation-function, such that

$$
E[X(u)] \neq 0 \quad \text { for some } u>0,
$$

then $\{X(t): t>0\}$ is a self-similar process.

Proof. Clearly, such a process has a spanning family of expectations so, by Theorem 2.1, its uniquely determined scaling family $\{A(t): t>0\}$ is a multiplicative group of operators. 
Keeping in mind that operators $T$ on the field of scalars are functions of the form $T(z)=$ $c z$, the operator norm $\|T\|$ being $|c|$, one can write

$$
|E[X(t u)]-E[X(u)]|=\left|(A(t)-I) \int_{\Omega} X(u) d P\right|=|| A(t)-I|||E[X(u)]| .
$$

The continuity of the expectation-function and the fact that $E[X(u)] \neq 0$ imply that the semigroup is uniformly continuous, hence it must be of the form

$$
A(t)=t^{H} I, \quad t>0
$$

for some scalar $H$ [7, Theorem 1.5, page 8], and hence the process is self-similar.

Of course, if $\{Y(t): t>0\}$ has zero expectations, but is obtained as in Remark 2.1 from an OSS process $\{X(t): t>0\}$ satisfying the assumptions in the corollary above, then $\{Y(t): t>0\}$ is self-similar. Another application of Theorem 2.1 is the following theorem where, under some assumptions, we have information about the growth of $\|E[X(t)]\|$ when $\{X(t): t>0\}$ is an OSS process.

Theorem 2.3. Let $\{X(t): t>0\}$ be an integrable OSS process with continuous expectationfunction. If Span $\{E[X(t)]: t>0\}$ is a subset having category 2 (in the sense of Baire) in the space $\mathscr{E}_{0}$, then there exist constants $M \geq 1$ and $a \geq 0$ such that

$$
\begin{gathered}
\|E[X(t)]\| \leq M t^{a}, \quad t \geq 1, \\
\|E[X(t)]\| \leq M t^{-a}, \quad 0<t \leq 1 .
\end{gathered}
$$

Proof. If $E[X(t)]=0$ for any $t>0$, the conclusion follows trivially. If there exists $u>0$ such that $E[X(u)] \neq 0$, then $\mathscr{E}_{0} \neq\{0\}$, and recall that $\mathscr{E}_{0}$ is left invariant by any scaling family of operators $\{A(t): t>0\}$. Denote by $B(t):=\left.A(t)\right|_{\mathscr{E}_{0}} \in \mathscr{L}\left(\mathscr{E}_{0}\right)$. By an argument similar to the one used in the proof of Theorem 2.1, $\{B(t): t>0\}$ is a multiplicative group of operators on $\mathscr{E}_{0}$.

Next we consider the multiplicative semigroup of operators obtained by taking indices in the interval $[1, \infty)$ and show that it is a multiplicative $C_{0}$-semigroup of operators, that is,

$$
\lim _{t \rightarrow 1^{+}} B(t) x=x
$$

for each $x$ in $\mathscr{E}_{0}$. Clearly, if one considers $x$ of the form $x=E[X(s)]$ for some $s>0$, the condition above holds because

$$
B(t) E[X(s)]=A(t) E[X(s)]=E[X(s t)]
$$

and the expectation-function is continuous. By linearity the same is true if one chooses $x$ in $\operatorname{Span}\{E[X(s)]: s>0\}$. To prove that (2.17) holds for any $x \in \mathscr{E}_{0}$, one needs to establish first the fact that the family $\{B(t): t>0\}$ is norm-bounded on a right neighborhood of 1 or, in other words, that there are some constants $M^{\prime}>0$ and $\delta>0$ such that

$$
\|B(u)\| \leq M^{\prime}, \quad \forall u \in(1,1+\delta) .
$$


For each $t>0,\{E[X(u)]: u \in[t, t(1+\delta)]\}$ is a compact set, hence a norm-bounded subset of $\mathscr{E}_{0}$. This implies that the set $\{B(u) E[X(t)]: u \in(1,1+\delta)\}$ is norm-bounded. By the linearity of the operators $B(u), u>0$, it readily follows that for each $x \in \operatorname{Span}\{E[X(t)]$ : $t>0\}$, the set $\{B(u) x: u \in(1,1+\delta)\}$ is norm-bounded. According to the Banach-Steinhaus theorem [16], this implies that the family $\{B(u): u \in(1,1+\delta)\}$ is norm-bounded. Based on that, the fact that property (2.17) holds for each $x \in \operatorname{Span}\{E[X(t)]: t>0\}$ extends to the fact that it holds for each $x \in \mathscr{E}_{0}$ by a straightforward argument. Indeed, let $\epsilon>0$ be arbitrary and fixed. Consider any fixed $x \in \mathscr{E}_{0}$. Since $\mathscr{E}_{0}$ is the closure of $\operatorname{Span}\{E[X(t)]: t>0\}$, we can choose $y \in \operatorname{Span}\{E[X(t)]: t>0\}$ such that $\|x-y\|<\epsilon / 3$ and $M^{\prime}\|x-y\|<\epsilon / 3$.

Also, since $B(t) y \rightarrow y$ as $t \rightarrow 1^{+}$we can choose $0<\delta_{1}<\delta$ such that $\|B(t) y-y\|<\epsilon / 3$ for all $t \in\left(1,1+\delta_{1}\right) \subset(1,1+\delta)$. Now

$$
\begin{aligned}
\|B(t) x-x\| & \leq\|B(t) x-B(t) y\|+\|B(t) y-y\|+\|y-x\| \\
& \leq\|B(t)\| \cdot\|x-y\|+\|B(t) y-y\|+\|y-x\| \\
& \leq M^{\prime}\|x-y\|+\|B(t) y-y\|+\|y-x\| \\
& \leq \frac{\epsilon}{3}+\frac{\epsilon}{3}+\frac{\epsilon}{3}=\epsilon
\end{aligned}
$$

for all $t \in\left(1,1+\delta_{1}\right)$, that is, $\lim _{t \rightarrow 1^{+}} B(t) x=x$ for all $x \in \mathscr{E}_{0}$.

Since $\{B(t): t \geq 1\}$ is a multiplicative $C_{0}$-semigroup of operators, there exist $M_{1} \geq 1$ and $a_{1} \geq 0$ such that

$$
\|B(s)\| \leq M_{1} s^{a_{1}}, \quad s \geq 1 .
$$

But $\{B(t): t>0\}$ is a multiplicative group of operators. According to [7, page 40], $\{V(s):=$ $\left.B\left(s^{-1}\right): s \geq 1\right\}$ is also a multiplicative $C_{0}$-semigroup of operators so there exist $M_{2} \geq 1$ and $a_{2} \geq 0$, such that

$$
\|B(s)\| \leq M_{2} s^{-a_{2}}, \quad s \in(0,1] .
$$

Thus, if $M_{0}=\max \left\{M_{1}, M_{2}\right\}$ and $a=\max \left\{a_{1}, a_{2}\right\}$, then

$$
\begin{gathered}
\|B(t)\| \leq M_{0} t^{a}, \quad t>1, \\
\|B(t)\| \leq M_{0} t^{-a}, \quad t \in(0,1] .
\end{gathered}
$$

Now

$$
\begin{aligned}
\|E[X(t)]\| & =\left\|\int_{\Omega} A(t) X(1) d P\right\|=\left\|B(t) \int_{\Omega} X(1) d P\right\| \\
& \leq\|B(t)\| \cdot\left\|\int_{\Omega} X(1) d P\right\| \leq M_{0} t^{a}\left\|\int_{\Omega} X(1) d P\right\|=M t^{a}
\end{aligned}
$$

for $t \geq 1$, where $M=M_{0}\left\|\int_{\Omega} X(1) d P\right\|$. 
If $t \in(0,1]$,

$$
\|E[X(t)]\| \leq\|B(t)\| \cdot\left\|\int_{\Omega} X(1) d P\right\| \leq M_{0} t^{-a}\left\|\int_{\Omega} X(1) d P\right\|=M t^{-a}
$$

Remark 2.3. Under the hypotheses above, if in addition, $\{X(t): t>0\}$ has a spanning family of expectations, then the conclusion can be strengthened to

$$
\begin{gathered}
E[\|X(t)\|] \leq M t^{a}, \quad t \geq 1, \\
E[\|X(t)\|] \leq M t^{-a}, \quad t \in(0,1] .
\end{gathered}
$$

Proof. Indeed, in this case $\mathscr{E}_{0}=\mathscr{E}$ and hence $B(t)=A(t)$, for all $t>0$. So

$$
\begin{aligned}
E[\|X(t)\|] & =\int_{\Omega}\|X(t)\| d P=\int_{\Omega}\|A(t) X(1)\| d P \\
& =\int_{\Omega}\|B(t) X(1)\| d P \leq \int_{\Omega}\|B(t)\| \cdot\|X(1)\| d P
\end{aligned}
$$

which in turn is less than or equal to

$$
M_{0} t^{a} \int_{\Omega}\|X(1)\| d P=M t^{a}, \quad \text { if } t \geq 1
$$

respectively,

$$
M_{0} t^{-a} \int_{\Omega}\|X(1)\| d P=M t^{-a}, \quad \text { if } t \in(0,1]
$$

where $M_{0}$ denotes the same constant as in the proof of the theorem above, and $M=$ $M_{0} \int_{\Omega}\|X(1)\| d P$.

Remark 2.4. If $\{X(t): t>0\}$ is an integrable OSS process with continuous expectationfunction and if $\operatorname{dim}\left(\mathscr{E}_{0}\right)<\infty$, then there exist constants $M \geq 1$ and $a \geq 0$ satisfying (2.16). In particular, if $\operatorname{dim}\left(\mathscr{E}_{0}\right)<\infty$ and $\mathscr{E}=\mathscr{E}_{0}$, then there exists $H \in \mathscr{L}(\mathscr{E})$ such that $A(t)=t^{H}$, for all $t>0$.

Proof. If $\operatorname{dim}\left(\mathscr{E}_{0}\right)<\infty$, then clearly $\operatorname{Span}\{E[X(t)]: t>0\}$ is a subset of $\mathscr{E}_{0}$ having category 2 because $\operatorname{Span}\{E[X(t)]: t>0\}=\mathscr{E}_{0}$. In the particular case $\mathscr{E}=\mathscr{E}_{0}, \operatorname{dim}\left(\mathscr{E}_{0}\right)<\infty$, according to the previous remark, $\{A(t): t \geq 1\}$ is a multiplicative $C_{0}$-semigroup of operators. Therefore $\{A(t): t \geq 1\}$ is a uniformly continuous multiplicative semigroup of operators, because $\operatorname{dim}(\mathscr{E})<\infty$. For this reason, there is an $H \in \mathscr{L}(\mathscr{E})$ such that $A(t)=t^{H}$, for all $t \geq 1$. But $\{A(t): t>0\}$ is a multiplicative group of operators. Therefore $A(1 / t)=$ $A(t)^{-1}$, for all $t \geq 1$, that is, $A(1 / t)=\left(t^{H}\right)^{-1}=(1 / t)^{H}$. If we denote $1 / t=s$, then $A(s)=s^{H}$, for all $s \in(0,1]$.

The existence of an $H$ as above can be obtained on infinite-dimensional spaces if one considers processes with the property that the expectation-function is differentiable on an interval of the form $\left(s_{0}, \infty\right)$ for some fixed $s_{0} \geq 1$. 
Theorem 2.4. If the integrable OSS process $\{X(t): t>0\}$ has the property that the expectation-function is differentiable on an interval of the form $\left(s_{0}, \infty\right)$ for some $s_{0} \geq 1$, $\mathscr{E}=\overline{\operatorname{Span}}\left\{E[X(s)]: s>s_{0}\right\}$, and $\operatorname{Span}\left\{E[X(s)]: s>s_{0}\right\}$ is a subset of $\mathscr{E}$ having category 2 in the sense of Baire, then the scaling family of operators of the process is of the form $A(s)=s^{H}$, $s>0$, where $H$ is a bounded linear operator on $\mathscr{E}$, uniquely determined by the following equation:

$$
H E[X(s)]=s \frac{d E[X(s)]}{d s} \quad \forall s>s_{0} .
$$

Proof. Observe that by Theorem 2.1, $\{A(t): t>0\}$ is a uniquely determined multiplicative group of operators. We need to show first that the family of operators $\{(A(v)-$ I) $/ \log v: v>0\}$ is norm-bounded for $v$ in the neighborhood of 1 , that is, for all $v \in$ $(1-\delta, 1+\delta)$, for some $\delta, 0<\delta<1$. As in the proof of Theorem 2.3, it will suffice to establish the pointwise boundedness of the family on a set having category 2 in the sense of Baire, namely, on $\operatorname{Span}\left\{E[(X(s))]: s>s_{0}\right\}$. For each $s>s_{0}$ one can write

$$
\lim _{v \rightarrow 1} \frac{(A(v)-I) E[X(s)]}{\log v}=\lim _{v \rightarrow 1} \frac{E[X(s v)]-E[X(s)]}{\log v}=s \frac{d(E[X(s)])}{d s} .
$$

Now this implies that for each $x \in \operatorname{Span}\left\{E[(X(s))]: s>s_{0}\right\}$ the family $\{(A(v)-I) x / \log v\}$ is bounded near 1 . On the other hand, the set $\{A(v) x: v \in[1,1+\delta)\}$ is also normbounded, by the continuity of the expectation-function on $\left(s_{0}, \infty\right)$. Arguing like in the proof of Theorem 2.3, one proves that $\{A(s): s>0\}$ is a multiplicative $C_{0}$-group of operators. Hence $\{(A(v)-I) x: v \in(1-\delta, 1+\delta)\}$ is norm-bounded and since $1 / \log v$ is bounded for $v$ in each set of the form $(1-\delta, 1-\epsilon) \cup(1+\epsilon, 1+\delta), 0<\epsilon<\delta$, one deduces that the set $\{(A(v)-I) x / \log v: v \in(1-\delta, 1+\delta)\}$ is norm-bounded for each $x$ in the space $\operatorname{Span}\left\{E[X(s)]: s>s_{0}\right\}$ which has category 2 in $\mathscr{E}$. By the Banach-Steinhaus theorem, there exists a constant $M \geq 0$ such that

$$
\frac{\| A(v)-I||}{|\log v|} \leq M, \quad \forall v \in(1-\delta, 1+\delta) .
$$

Since $\{A(t): t>0\}$ is a multiplicative $C_{0}$-group of operators, $\left\{T(t)=A\left(e^{t}\right): t \in \mathbb{R}\right\}$ is an additive $C_{0}$-group of operators. Setting $t_{0}=\log s_{0}$ we wish to show that for each $x \in \mathscr{E}$, the function $T(\cdot) x$ is differentiable at $t$ for any $t>t_{0}$. We begin by showing this fact for $x$ of the form $x=E[X(u)], u \geq 1$. Indeed, in such a case, the following limit exists for any $s>s_{0}$ :

$$
\lim _{v \rightarrow 1} \frac{(A(s v)-A(s))(E[X(u)])}{\log v}=s u \frac{d E[X(s u)]}{d t} .
$$

Taking $s=e^{t}$ and making the substitution $h=\log v$, we obtain that the following limit exists, for any $u \geq 1$ and hence for any $u>s_{0}$ :

$$
\lim _{h \rightarrow 0} \frac{(T(t+h)-T(t)) E[X(u)]}{h} .
$$


By linearity, this implies that for any $t>t_{0}$ and any $x \in \operatorname{Span}\left(\left\{E[X(u)]: u>s_{0}\right\}\right)$, the function $T(\cdot) x$ is differentiable at $t$. To prove this for an arbitrary $x \in \mathscr{E}$, observe that the inequality

$$
\frac{\| A(v)-I||}{|\log v|} \leq M, \quad \forall v \in(1-\delta, 1+\delta),
$$

implies

$$
\frac{\|T(h)-I\|}{|h|} \leq M, \quad \forall h \in\left(-\delta^{\prime}, \delta^{\prime}\right),
$$

where $\delta^{\prime}>0$ is chosen such that $\left(e^{-\delta^{\prime}}, e^{\delta^{\prime}}\right) \subseteq(1-\delta, 1+\delta)$. For an arbitrary sequence $\left\{h_{n}\right\}_{n}$ in $\mathbb{R}$ such that $h_{n} \rightarrow 0$, we wish to show that the sequence $\left\{\left(T\left(t+h_{n}\right)-T(t)\right) x / h_{n}\right\}_{n}$ is Cauchy. To that aim, choose $\epsilon>0$ and $y \in \operatorname{Span}\left(\left\{E[X(u)]: u>s_{0}\right\}\right)$ such that $\| x-$ $y \| \leq \epsilon /(4 M\|T(t)\|)$. Since the sequence $\left\{\left(T\left(t+h_{n}\right)-T(t)\right) y / h_{n}\right\}_{n}$ is Cauchy because it is convergent, we can choose $n_{0} \in \mathbb{N}$ such that

$$
\left\|\frac{\left(T\left(t+h_{m}\right)-T(t)\right) y}{h_{m}}-\frac{\left(T\left(t+h_{n}\right)-T(t)\right) y}{h_{n}}\right\|<\frac{\epsilon}{2}, \quad\left|h_{n}\right|<\delta^{\prime},
$$

for all $m, n \geq n_{0}$. In that case we can write

$$
\begin{aligned}
\left\|\frac{\left(T\left(t+h_{m}\right)-T(t)\right) x}{h_{m}}-\frac{\left(T\left(t+h_{n}\right)-T(t)\right) x}{h_{n}}\right\| \\
\leq\left\|\frac{\|\left(T\left(t+h_{m}\right)-T(t)\right) y}{h_{m}}-\frac{\left(T\left(t+h_{n}\right)-T(t)\right) y}{h_{n}}\right\| \\
\quad+\|T(t)\|\left(\frac{\left\|T\left(h_{m}\right)-I\right\|}{\left|h_{m}\right|}+\frac{\left\|T\left(h_{n}\right)-I\right\|}{\left|h_{n}\right|}\right)\|x-y\| \\
<\frac{\epsilon}{2}+\|T(t)\| 2 M \frac{\epsilon}{4 M\|T(t)\|}=\epsilon \quad \forall m, n \geq n_{0} .
\end{aligned}
$$

This shows that the derivative of the function $T(\cdot) x$ exists at any $t>t_{0}$ for any choice of $x \in \mathscr{E}$.

According to [14, Chapter 2, Lemma 4.2], this implies that, for $t>t_{0}$ sufficiently large, $H T(t)$ is a bounded operator, where $H$ is the infinitesimal generator of $\{T(t): t \in \mathbb{R}\}$. Then $H T(t) T(-t)=H$ is also bounded. Hence we have that $T(t)=e^{t H}$, for all $t \in \mathbb{R}$, that is, $A(s)=s^{H}$, for all $s>0$. To show that $H$ satisfies (2.30), observe that for each fixed $s>s_{0}$, one can write

$$
\begin{aligned}
H E[X(s)] & =\lim _{t \rightarrow 0^{+}} \frac{T(t) E[X(s)]-E[X(s)]}{t} \\
& =\lim _{v \rightarrow 1^{+}} \frac{(A(v)-I) E[X(s)]}{\log v}=\lim _{v \rightarrow 1^{+}} \frac{E[X(s v)]-E[X(s)]}{\log v}=s \frac{d(E[X(s)])}{d s} .
\end{aligned}
$$




\section{OSS processes with scaling families of invertible operators}

In this section we assume that for the OSS process $\{X(t): t>0\}$ there exists a scaling family of operators $\{A(s): s>0\}$ consisting of invertible operators on $\mathscr{E}$. For such processes we consider the classes of operators in the following definition.

Definition 3.1. For each arbitrary, fixed $s>0 G_{s}$, is by definition the class of all invertible operators $A \in \mathscr{L}(\mathscr{E})$ with the property

$$
\begin{gathered}
\{A X(t)\} \stackrel{d}{=}\{X(s t)\}, \\
G=\bigcup_{t>0} G_{t} .
\end{gathered}
$$

Remark 3.2. By our assumptions $G_{t} \neq \varnothing$, for any $t>0$.

The following theorem (parts of which appear in [4] or [17] for the case of operators on $\mathbb{R}^{n}$ ) has a straightforward proof. We include it in order to make this paper selfcontained.

Theorem 3.1. G is a group, $G_{1}$ is a normal subgroup of $G$, closed relative to $G$. For each $t>0$, the class $G_{t}$ is an equivalence class modulo $G_{1}$, that is, $G_{t} \in G / G_{1}$, for any $t>0$, and the map $\varphi(t)=G_{t}, t>0$, is an onto group homomorphism of $(0, \infty)$ onto $G / G_{1}$. Let $p$ denote the canonical projection of $G$ onto $G / G_{1}$. The process $\{X(t): t>0\}$ has a scaling family of operators which is a multiplicative group of operators if and only if the homomorphism $\varphi$ lifts to a homomorphism $\psi$ of $(0, \infty)$ into $G$, that is, if and only if there exists a group homomorphism $\psi$ of the multiplicative group $(0, \infty)$ into $G$ such that $p \circ \psi=\varphi$.

Proof. Indeed, for $u, s>0$ consider $A \in G_{s}$ and $B \in G_{u}$. We can write $\{B X(t)\} \stackrel{d}{=}\{X(u t)\}$, hence $\{X(t)\} \stackrel{d}{=}\left\{B^{-1} X(u t)\right\}$. Denote now $t u=v$, hence $t=u^{-1} v$ and we get $\left\{B^{-1} X(v)\right\} \stackrel{d}{=}$ $\left\{X\left(u^{-1} v\right)\right\}$. This proves that $B^{-1} \in G_{u^{-1}}$ if $B \in G_{u}$, that is, inverses of operators in $G$ belong to $G$. Now $\{B X(t)\} \stackrel{d}{=}\{X(u t)\}$ and $\{A X(t)\} \stackrel{d}{=}\{X(s t)\}$. Therefore $\{A B X(t)\} \stackrel{d}{=}$ $\{A X(u t)\} \stackrel{d}{=}\{X(s u t)\}$, that is, $A B \in G_{s u}$ which proves that $G$ is a subgroup of the group of all invertible operators on $\mathscr{E}$ and that $\varphi$ is an onto group homomorphism, provided that we show that the sets $G_{s}, s>0$ are equivalence classes modulo $G_{1}$. We will prove the latter below. In a similar way, one shows that $G_{1}$ is a subgroup of $G$. Let us check that $G_{1}$ is normal. Indeed, choose an arbitrary $A$ in $G$. Then $A \in G_{s}$ for some $s>0$. For any $B \in G_{1}$ we can write

$$
\begin{gathered}
\{B X(t)\} \stackrel{d}{=}\{X(t)\}, \\
\{A X(t)\} \stackrel{d}{=}\{X(s t)\} .
\end{gathered}
$$

So

$$
\{A B X(t)\} \stackrel{d}{=}\{A X(t)\} \stackrel{d}{=}\{X(s t)\}
$$

that is, $A B \in G_{s}$ or $A G_{1} \subseteq G_{s}$. 
Conversely now, if $T \in G_{s}$, then set $B=A^{-1} T$. According to what we proved above, $B \in G_{1}$ and $A B=T \in A G_{1}$, proving that $A G_{1}=G_{s}$. A similar argument leads to $G_{1} A=$ $G_{s}$, ending the proof of the normality of $G_{1}$ and of the fact that the sets $G_{s}$ are equivalence classes modulo $G_{1}$. Finally the fact that $G_{1}$ is closed relative to $G$ (not only norm-closed but closed even with respect to the strong operator topology) is a direct consequence of $[6$, Proposition 1.7.2]. Obviously, the process $\{X(t): t>0\}$ has a scaling family of operators which is a multiplicative group of operators if and only if the homomorphism $\varphi$ lifts to a homomorphism $\psi$ of $(0, \infty)$ into $G$.

In several of the previous papers on OSS processes $[4,9,17]$, it is proved that such processes satisfying good continuity assumptions have an exponent, that is, have a scaling family of operators of the form $\left\{s^{H}: s>0\right\}$ where $H \in \mathscr{L}(\mathscr{E})$ is called an exponent of the process. Relative to that, we prove the following.

Theorem 3.2. The OSS process $\{X(t): t>0\}$ has an exponent if and only if there exists a locally compact, Abelian subgroup $A$ of $G$ with the following properties:

$$
\begin{gathered}
\widetilde{G_{s}}:=\mathscr{A} \cap G_{s} \neq \varnothing \quad \forall s>0, \\
\forall \epsilon>0 \quad \exists(0<\delta<1) \text { such that if }|t-1|<\delta, \text { then } \\
\exists A \in \widetilde{G_{t}} \text { such that }\|A-I\|<\epsilon .
\end{gathered}
$$

Proof. To prove the sufficiency observe first that $\widetilde{G_{1}}$ is a subgroup of $\mathscr{A}$ closed relative to $\mathscr{A}$, for each $s>0 \widetilde{G_{s}}$ is an equivalence class of $\mathscr{A}$, modulo $\widetilde{G_{1}}$, and $\varphi(s)=\widetilde{G_{s}}$ is an onto group homomorphism of $(0, \infty)$ onto $\mathscr{A} / \widetilde{G_{1}}$. This homomorphism is continuous if the quotient topology is considered on $\mathscr{A} / \widetilde{G_{1}}$. Since this is a homomorphism of topological groups, only continuity at 1 needs to be checked. To that aim, let $\mathcal{N}$ denote a neighborhood of the identity in $\mathscr{A} / \widetilde{G_{1}}$. In that case there exist $S \subseteq(0, \infty)$ such that $1 \in S$, $\mathcal{N}=\left\{\widetilde{G_{s}}: s \in S\right\}$, and $N=\cup_{s \in S} \widetilde{G_{s}}$ is a neighborhood of $I$. Therefore there is an $\epsilon>0$ such that, if $A \in \mathscr{A}$ and $\|A-I\|<\epsilon$, then $A \in N$. Associate to this $\epsilon$ a $\delta$ satisfying condition (3.5). For arbitrary, fixed $t \in(1-\delta, 1+\delta)$ consider an operator $A$ as in condition (3.5). There must exist $s \in S$ such that $A \in \widetilde{G_{s}}$ and hence $\widetilde{G_{t}}=\widetilde{G_{s}}$ so $\widetilde{G_{t}} \in \mathcal{N}$, or in other words, $\varphi(1-\delta, 1+\delta) \subseteq \mathcal{N}$, that is, $\varphi$ is continuous. By a theorem of Moskowitz (see [1] or [13]), $\varphi$ lifts to a continuous group homomorphism $\psi:(0, \infty) \rightarrow \mathscr{A}$, hence $\{A(t)=\psi(t): t>0\}$ is both a scaling family for the process under consideration and a norm-continuous multiplicative group of operators. Therefore it must be of the form $\left\{A(s)=s^{H}: s>0\right\}$ for some $H \in \mathscr{L}(\mathscr{E})$, which ends the proof of the sufficiency. The necessity is immediate. Indeed if an exponent $H$ exists, then set $\mathscr{A}=\left\{s^{H}: s>0\right\}$.

Exponents and more generally, scaling families of operators for OSS processes need not be uniquely determined. See $[4,9,17]$ or the following remark.

Remark 3.3. Let $A$ be an arbitrary operator in $G$ and $\rho_{A}$ the inner automorphism of $G$ induced by $A$,

$$
\rho_{A}(T)=A^{-1} T A, \quad T \in G
$$


The image under $\rho_{A}$ of any scaling family of operators of the process $\{X(t): t>0\}$ is also a scaling family of operators for the same process.

Proof. Assume $A \in G_{s}$ for some $s>0$, then let $\{A(t): t>0\}$ be a scaling family of operators for the given process. By the proof of Theorem 3.1, $A^{-1} \in G_{s^{-1}}$ and

$$
\rho_{A}(A(t))=A^{-1} A(t) A \in G_{s^{-1} t s}=G_{t} \quad \forall t>0 .
$$

Next we will extend to arbitrary Hilbert spaces the early result by Laha and Rohatgi saying that proper $\mathbb{R}^{n}$-valued OSS processes with a scaling family of positive operators have exponents. Let $\mathscr{E}$ be a Hilbert space. We need to introduce some terminology first.

Definition 3.4. Recall that a probability measure $\mu$ on the $\sigma$-algebra of the Borel subsets of $\mathscr{E}$, a separable Banach space, is called a full measure if the support of $\mu$ is not contained by a hyperplane.

Following [9], we introduce the notion of proper stochastic process.

Definition 3.5. The process $\{X(t): t>0\}$ is called proper if $P X(t)^{-1}$ is a full probability measure for any $t>0$.

Remark 3.6. An OSS process $\{X(t): t>0\}$ with a scaling family of invertible operators is proper if and only if $P X(1)^{-1}$ is a full probability measure.

Proof. Indeed, this is a consequence of the equality

$$
\operatorname{supp}\left[P X(t)^{-1}\right]=A(t)\left(\operatorname{supp}\left[P X(1)^{-1}\right]\right) .
$$

Lemma 3.3. If $\mathscr{E}$ is a separable Hilbert space and $A \in \mathscr{L}(\mathscr{E})$ is a positive invertible operator other than the identity, then there exists a nonzero vector $x_{0} \in \mathscr{E}$ such that either $\left\|A^{n} x_{0}\right\| \rightarrow 0$ or $\left\|A^{-n} x_{0}\right\| \rightarrow 0$. If $A$ is positive and noninvertible, then there exists some nonzero $x_{0}$ such that $\left\|A^{n} x_{0}\right\| \rightarrow 0$.

Proof. Assume first that $A$ is invertible and $A \neq I$. By the spectral mapping theorem for positive operators, [15, Theorem 1.6] it will be enough to prove the existence of $x_{0}$ for a multiplication operator $M_{\phi}$, acting on $L_{K}^{2}(d \mu)$ where $K$ is a compact subset of $(0, \infty), \mu$ a finite Borel measure on $K$, and $\phi$ an essentially bounded nonnegative function on $K$ with essentially bounded inverse $\phi^{-1}$. Indeed, each positive invertible operator $A$ is unitarily equivalent to such a multiplication operator $M_{\phi}$ by [15, Theorem 1.6]. If the set $E=\{x \in K: \phi(x)<1\}$ has positive measure $\mu$, then its characteristic function $\chi_{E}$ is a nonzero element of $L_{K}^{2}(d \mu)$. By Lebesgue's dominated convergence theorem, one immediately proves $\left\|M_{\phi}^{n} \chi_{E}\right\|_{2} \rightarrow 0$. If $\mu(E)=0$, then suppose $\mu(F)>0$, where $F=\left\{x \in K: \phi^{-1}(x)<1\right\}$. Again by Lebesgue's theorem, one gets $\left\|M_{\phi}^{-n} \chi_{F}\right\|_{2} \rightarrow 0$. One of the sets $E$ and $F$ must have positive measure $\mu$ because if we suppose that both have measure 0 , then $\phi=1, \mu$ - a.e., that is, $M_{\phi}=I$ and hence $A=I$, contrary to our assumptions. Thus we established the existence of a nonzero $x_{0}$ with the required properties. To show the existence of $x_{0}$ when $A$ is noninvertible, recall that $K$ above is the spectrum of $A$ and in this last case $K$ contains 0 . It is known that $K$ coincides with the essential range of $\phi$, 
[3]. Let us choose $\epsilon, 0<\epsilon<1$. Since 0 is in the essential range of $\phi$, one obtains that the set $E=\phi^{-1}([0, \epsilon))$ has positive measure $\mu$. Therefore $\chi_{E}$ is a nonzero element of $L_{K}^{2}(d \mu)$, and since $\phi^{n}(x) \rightarrow 0$, for all $x \in E$, Lebesgue's theorem can be applied again in order to get $\left\|M_{\phi}^{n} \chi_{E}\right\|_{2} \rightarrow 0$.

Lemma 3.4. Let $\mathscr{E}$ be a separable Hilbert space and $X(1): \Omega \rightarrow \mathscr{E}$ a random variable such that $P X(1)^{-1}$ is a full probability measure. The only positive operator $A$ on $\mathscr{E}$ with the property $P(A X(1))^{-1}=P(X(1))^{-1}$ is the identity.

Proof. If $A$ is invertible then obviously $P\left(A^{-1} X(1)\right)^{-1}=P(X(1))^{-1}$. Therefore we will not reduce generality by assuming that $\left\|A^{n} x_{0}\right\| \rightarrow 0$ for some nonzero $x_{0}$. Denote by $\langle\cdot, \cdot\rangle$ the inner product of $\mathscr{E}$. The mapping $\left\langle\cdot, x_{0}\right\rangle$ is obviously a continuous mapping on $\mathscr{E}$. Therefore one can write

$$
P\left(\left\langle X(1), x_{0}\right\rangle\right)^{-1}=P\left(\left\langle A^{n} X(1), x_{0}\right\rangle\right)^{-1}=P\left(\left\langle X(1), A^{n} x_{0}\right\rangle\right)^{-1}, \quad \forall n \geq 0 .
$$

Since $\left\|A^{n} x_{0}\right\| \rightarrow 0$, one deduces $P\left(\left\langle X(1), x_{0}\right\rangle\right)^{-1}=\delta_{0}$. By [6, page 27]

$$
\left\langle\operatorname{supp}\left[P X(1)^{-1}\right], x_{0}\right\rangle=\{0\}=\operatorname{supp}\left[\delta_{0}\right]
$$

hence

$$
\overline{\operatorname{Span}}\left(\operatorname{supp}\left[P X(1)^{-1}\right]\right) \subseteq \mathscr{E} \ominus \operatorname{Span}\left\{x_{0}\right\}
$$

which is a contradiction because this means that $P X(1)^{-1}$ is not full.

TheOREm 3.5. If $\mathscr{E}$ is a separable Hilbert space and $\{X(t): t>0\}$ is an OSS process with a scaling family $\{A(t): t>0\}$ of positive operators, commuting with each other, and if $P X(1)^{-1}$ is full, then the process is proper and $\{A(t): t>0\}$ is a multiplicative group of operators.

Proof. By Lemma 3.4, $G_{1}$ contains only one positive operator, namely, I. This implies $A(1)=I$. For any $t>0 A(t)$ and $A\left(t^{-1}\right)$ are commuting positive operators, hence $A(t) A\left(t^{-1}\right)$ is positive. Since $A(t) A\left(t^{-1}\right) \in G_{1}$, one deduces $A(t) A\left(t^{-1}\right)=I$. This proves that the scaling family of operators consists of invertible positive operators. By Remark 3.6 this fact implies that the process under consideration is proper. Finally, in order to establish the fact that the scaling family of operators is a multiplicative group, choose $s, t>0$ arbitrary but fixed and observe that $A(s), A(t)$, and $A(s t)$ are positive operators commuting with each other, for which reason $A^{-1}(s t) A(s) A(t)$ is a positive operator in $G_{1}$ hence $A(s t)=A(s) A(t)$.

\section{References}

[1] D. L. Armacost, The Structure of Locally Compact Abelian Groups, Monographs and Textbooks in Pure and Applied Mathematics, vol. 68, Marcel Dekker, New York, 1981.

[2] R. B. Ash, Real Analysis and Probability, Academic Press, New York, 1972.

[3] P. R. Halmos, A Hilbert Space Problem Book, D. Van Nostrand, New Jersey, 1967.

[4] W. N. Hudson and J. D. Mason, Operator-self-similar processes in a finite-dimensional space, Transactions of the American Mathematical Society 273 (1982), no. 1, 281-297. 
[5] Z. J. Jurek, Limit distributions and one-parameter groups of linear operators on Banach spaces, Journal of Multivariate Analysis 13 (1983), no. 4, 578-604.

[6] Z. J. Jurek and J. D. Mason, Operator-Limit Distributions in Probability Theory, Wiley Series in Probability and Mathematical Statistics: Probability and Mathematical Statistics, John Wiley \& Sons, New York, 1993.

[7] S. Kantorovitz, Semigroups of Operators and Spectral Theory, Pitman Research Notes in Mathematics Series, vol. 330, Longman Scientific \& Technical, Harlow, 1995.

[8] W. Krakowiak, Operator-stable probability measures on Banach spaces, Colloquium Mathematicum 41 (1979), no. 2, 313-326.

[9] R. G. Laha and V. K. Rohatgi, Operator self-similar processes, Zeitschrift für Wahrscheinlichkeitstheorie und Verwandte Gebiete 50 (1979), 5-25.

[10] J. Lamperti, Semi-stable stochastic processes, Transactions of the American Mathematical Society 104 (1962), 62-78.

[11] V. Mandrekar and M. M. Meerschaert, Sample moments and symmetric statistics, Stochastic Analysis on Infinite-Dimensional Spaces (Baton Rouge, La, 1994), Pitman Res. Notes Math. Ser., vol. 310, Longman Scientific \& Technical, Harlow, 1994, pp. 197-210.

[12] M. M. Meerschaert and H.-P. Scheffler, Limit Distributions for Sums of Independent Random Vectors. Heavy Tails in Theory and Practice, Wiley Series in Probability and Statistics: Probability and Statistics, John Wiley \& Sons, New York, 2001.

[13] M. Moskowitz, Homological algebra in locally compact abelian groups, Transactions of the American Mathematical Society 127 (1967), 361-404.

[14] A. Pazy, Semigroups of Linear Operators and Applications to Partial Differential Equations, Applied Mathematical Sciences, vol. 44, Springer, New York, 1983.

[15] H. Radjavi and P. Rosenthal, Invariant Subspaces, Springer, New York, 1973.

[16] W. Rudin, Functional Analysis, McGraw-Hill, New York, 1973.

[17] K. Sato, Self-similar processes with independent increments, Probability Theory and Related Fields 89 (1991), no. 3, 285-300.

[18] G. Siegel, Exponents of operator stable distributions in Banach spaces, Probability Theory and Mathematical Statistics, Vol. II (Vilnius, 1989), “Mokslas", Vilnius, 1990, pp. 437-445.

[19] - On the class of operator stable distributions in a separable Banach space, Probability and Mathematical Statistics 13 (1992), no. 1, 33-37.

[20] K. Urbanik, Lévy's probability measures on Banach spaces, Studia Mathematica 63 (1978), no. 3, 283-308.

Mihaela T. Matache: Department of Mathematics, University of Nebraska, Omaha,

NE 68182-0243, USA

E-mail address: dmatache@mail.unomaha.edu

Valentin Matache: Department of Mathematics, University of Nebraska, Omaha, NE 68182-0243, USA

E-mail address: vmatache@mail.unomaha.edu 


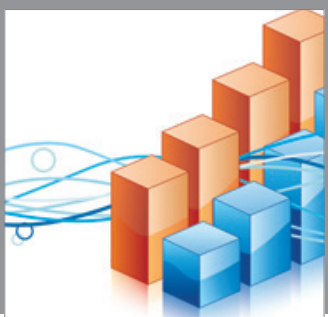

Advances in

Operations Research

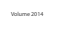

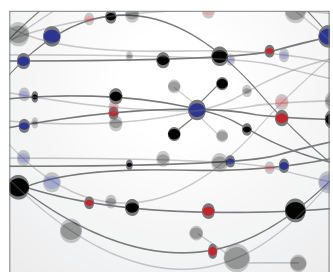

\section{The Scientific} World Journal
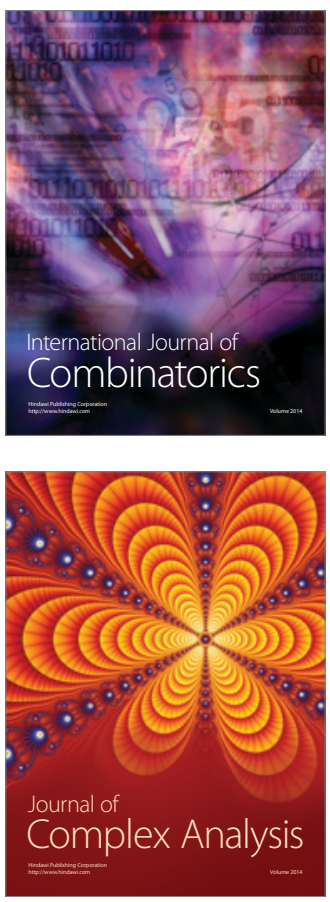

International Journal of

Mathematics and

Mathematical

Sciences
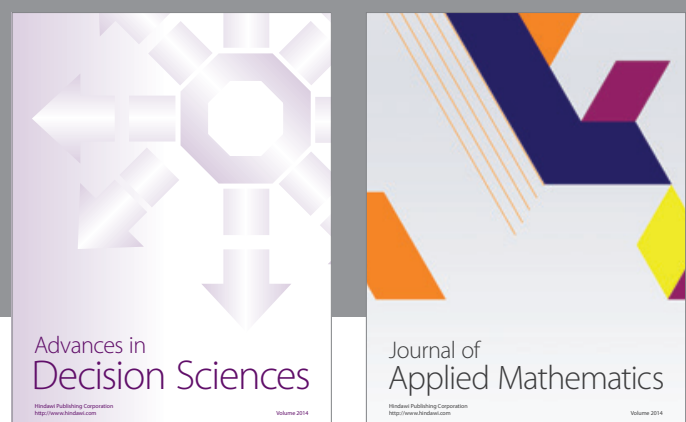

Journal of

Applied Mathematics
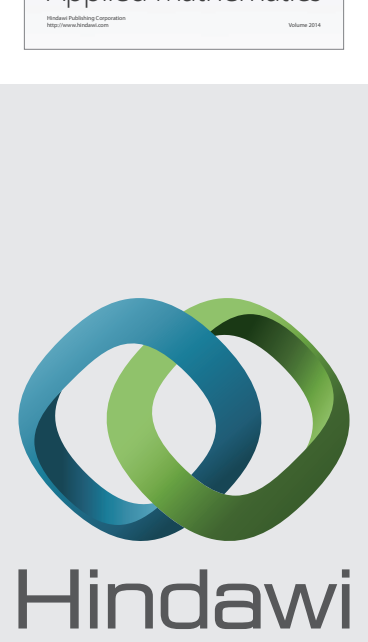

Submit your manuscripts at http://www.hindawi.com
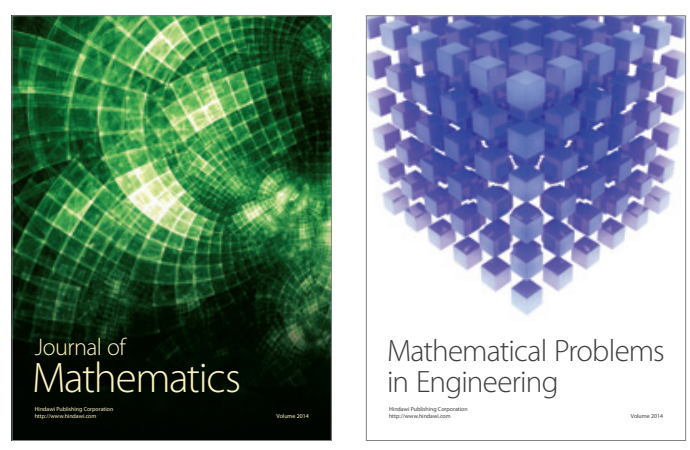

Mathematical Problems in Engineering
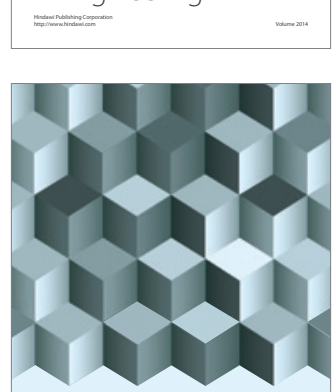

Journal of

Function Spaces
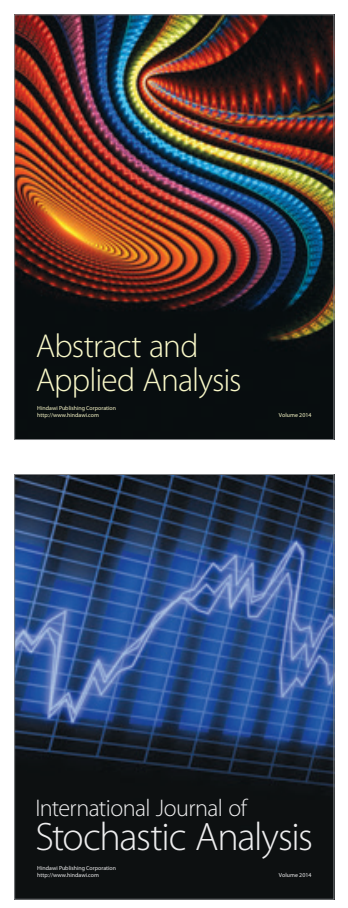

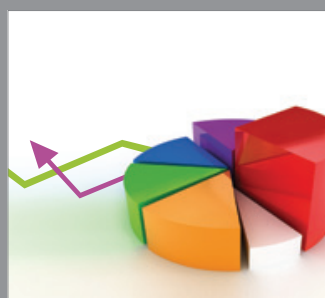

ournal of

Probability and Statistics

Promensencen
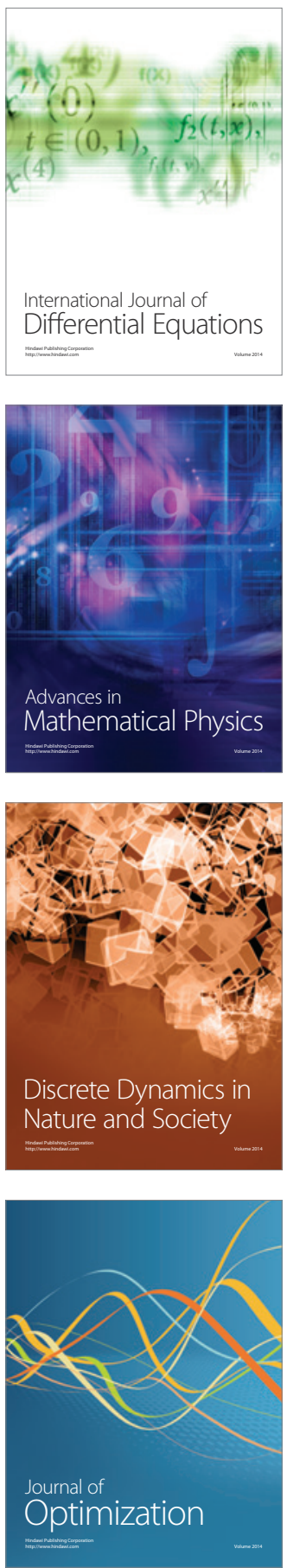